\title{
Urgences
}

\section{A. Superficies du domaine}

\section{André Gervais et Renald Bérubé}

Numéro 19, janvier 1988

Le tour du texte

URI : https://id.erudit.org/iderudit/025442ar

DOI : https://doi.org/10.7202/025442ar

Aller au sommaire du numéro

Éditeur(s)

Urgences

ISSN

0226-9554 (imprimé)

1927-3924 (numérique)

Découvrir la revue

Citer ce document

Gervais, A. \& Bérubé, R. (1988). A. Superficies du domaine. Urgences, (19),

14-26. https://doi.org/10.7202/025442ar d'utilisation que vous pouvez consulter en ligne.

https://apropos.erudit.org/fr/usagers/politique-dutilisation/ 


\section{A. Superficies du domaine:}

infratexte, infratextualité

ultratexte, ultratextualité

génotexte

phénotexte

avant-texte

après-texte

transtextualité

transtextuel

architexte, architextualité, architexture

paratexte, paratextualití

in-texte

hors-texte

extratexte, extratextualité 
RÉF. — Jean-Pierre Vidal, 1975 - «Le souverain s'avarie. Lecture de l'onomastique R-G». Robbe-Grillet. Colloque de Cerisy ftenu en 1975], tome I. Coll. «10/18», 1079, Paris, UGE, 1976, p. 273-309.

DÉF. - «Tout ce qui, sous la surface et dans les marges du texte manifeste le "manque à sa place" d'un signifiant ou d'une chaîne signifiante, génératrice et générée, produite par une absence ou un écart, et produisant des palimpsestes, comme autant de surcharges sémantiques. Filigrane, * intra et *intertexte donc, c'est un "pli» discursif qui feuillette lespace de la signifiance en "* phénotexte» et «* génotexte», pour reprendre ici le vocabulaire de Julia Kristeva.» Jean-Pierre Vidal: "L'infratexte. mode du génotexte ou fantasme de lecture". La Nouvelle Barre du jour. Montréal, no 103 (no intitulé L'infratexte), mai 1981, p. 29.

EX. - L'article ici cité, dans lequel sont analysés des textes de $M$. Duchamp, P. Verlaine, S. Mallarmé, Lautréamont et D. Diderot.

\section{Ultratexte, ultratextualité}

RÉF. - Jean-Pierre Vidal, 1981

DÉF.: - «De l'* infratexte à l'ultratexte, aussi (enfin), comme de l'infrarouge à l'ultraviolet, comme de l'infra-son à l'ultra-son. Spectre textuel, comme spectre solaire et sonore.»

André Gervais. Jean-Pierre Vidal et Ghislain Bourque: «Propositions initiales». La Nourelle Barre du jour. Montréal, no 103 (no intitulé L'infratexte), mai 1981, p. 14.

«Mais avant que lhallali ne se sonne quelque part dans les sous-bois $\mathrm{du}^{*}$ hors-texte, il est peut-être bon d'insister sur une autre conséquence de $l^{* *}$ infratexte, dans le champ cette fois des diverses théories du récit (Propp, Bremond, Genette, etc...) auxquelles on pourrait peut-être reprocher certain traitement du texte comme mythe, puisque récit sans autre signifiant, sans autre matériau que ses articulations diégétiques. En montrant que des fictions peuvent être aussi le produit d'une structuration dans les marges (...), la lecture infratextuelle pose le problème de l'autonomie relative du récit et rend fondamentale la question des rapports chaîne signifiante-chaîne diégétique. Si le récit, en ce sens, était un ultratexte?

$\mid$... $\mid$ ce projet majeur: la lecture dans toute sa bande, dans tout son spectre, de l'* infratextualité à l'ultratextualité, de ces Chants de Maldoror toujours encore déjà devant derrière tout autour de moi, de nous." 
Jean-Pierre Vidal: "Linfratexte, mode du génotexte ou fantasme de lecture". La Nouvelle Barre du jour: Montréal, no 103 (no intitulé $L$ infratexte), mai 1981. p. 51-52.

EX. - Le dernier article ici cité analyse (p. 44-46) tel épisode d'un roman de D. Diderot.

\section{Génotexte}

\section{RÉF. - Julia Kristeva, 1969}

DÉF. - «Premièrement, on doit faire une distinction radicale entre notre séparation géno-texte $/{ }^{*}$ phéno-texte et celle qu introduit la grammaire générative de Chomsky entre structure profonde et structure de surface. [...]

Ce que nous appelons un géno-texte est un niveau abstrait du fonctionnement linguistique qui, loin de refléter les structures de la phrase, et en précédant et excédant ces structures, fait leur anamnèse. Il s'agit donc d'un fonctionnement signifiant qui, tout en se faisant dans la langue, n'est pas réductible à la parole manifestée dans la communication dite normale (à ses universaux et aux lois de leur combinaison). Le géno-texte opère avec des catégories analytico-linguistiques (pour lesquelles nous devrions trouver à chaque fois dans le discours théorique des concepts analytico-linguistiques) dont la limite n'est pas de générer pour le * phéno-texte une phrase (sujet-prédicat), mais un signifiant pris à différents stades du processus du fonctionnement signifiant. Cette séquence peut être dans le * phéno-texte un mot, une suite de mots, une phrase nominale, un paragraphe, un "non-sens" etc.

Le géno-texte n'est pas une structure, mais il ne saurait être le structurant non plus, puisqu'il n'est pas ce qui forme ni ce qui permet à la structure d'être, fût-ce en restant censuré. Le géno-texte est le signifiant infini qui ne pourrait «être» un «ce» car il n'est pas un singulier; on le désignerait mieux comme «les signifiants" pluriels et différenciés à l’infini, par rapport auxquels le signifiant ici présent, le signifiant de la-formule-présente-du-sujet-dit n'est qu'une borne, un lieu-dit, une ac-cidence (c'est-à-dire un abord, une approximation qui s'ajoute aux signifiants en abandonnant sa position). Pluralité des signifiants dans laquelle - et non pas en dehors de laquelle - le signifiant formulé (du* phéno-texte) est situable et, comme tel, surdéterminé. Le géno-texte est ainsi non pas l'autre scène par rapport au présent formulaire et axial, mais l'ensemble des autres scènes dans la multiplicité desquelles il manque un index écarté écartelé — par la surdétermination qui définit, de l'intérieur, l'infini. [...]

Non structuré et non structurant, le géno-texte ne connait pas le 
sujet. Extérieur au sujet, il n'est même pas son négatif nihiliste, car il est son autre oeuvrant en deça et au-delà de lui. Lieu hors-subjectif et hors-temporel (le sujet et le temps n'apparaissent que comme des accidents de ce vaste fonctionnement qui les traverse), le géno-texte peut être présenté comme le dispositif de l'histoire de la langue et des pratiques signifiantes qu'elle est susceptible de connaitre: les possibilités de toutes les langues concrètes existantes et à venir y sont "données" avant de retomber masquées ou censurées dans le * phéno-texte.

Ces approximations concernant la distinction géno-texte/ * phéno-texte se rapprochent des théories linguistiques générativistes de Saumjan-Soboleva auxquelles les termes eux-mêmes sont empruntés."

Julia Kristeva: Sèméiôtikè. Recherches pour une sémanalyse. Coll. «Tel Quel», Paris, Seuil. 1969. p. 281-284.

EX. - Gérard Genette: "Métonymie chez Proust, ou la naissance du récit» [Poétique, Paris, no 2, 1970], repris sous l'intitulé "Métonymie chez Proust» dans Figures III. Coll. «Poétique», Paris, Seuil, 1972, p. 41-63.

\section{Phénotexte}

RÉF. - Julia Kristeva, 1969

DÉF.: - « (...) la distinction entre les deux termes * géno-texte/phénotexte si elle est purement didactique n'en procède pas moins d'une démarche matérialiste qui pose le principe de la structuration dans la matière même du structuré, démarche adoptée par un discours qui se veut théorique et qui essaie de penser une production textuelle qui, elle, vit constamment sur le devenir de la génération en formule, de l'engendrement en semence, et dans leur réfraction réciproque qui tisse le texte. (...)

Penser le phéno-texte comme une formule exige qu'on ouvre en lui un couloir de réminiscence double - à la fois vers le processus symbolique/mathématique que la signifiance textuelle rejoint en le pratiquant dans la langue, et vers le corpus idéologique/mythique qui sature chaque bloc de l'histoire monumentale.

[... Il est évident que ciest le sujet du discours théorique qui perce la surface phénoménologique de l'énoncé (le phéno-texte) qui est sa propre surface de description (...). Autrement dit, le sujet du discours théorique, qui aurait pu signorer dans une description phénoménologique, continue de s'ignorer $[. .$.$] tout en se reconsti-$ tuant dans un discours non soumis à une description objectale: c'est 
le discours qui pose le ${ }^{*}$ géno-texte. Le dédoublement du texte en * géno-texte et en phéno-texte n'est au fond qu un dédoublement du discours théorique."

Julia Kristeva: Sèméiotikè. Recherches pour une sémanalyse. Coll. "Tel Quel», Paris. Seuil. 1969. p. 285-288.

\section{Avant-texte}

RÉF. — Jean Bellemin-Noël, 1972

DÉF. _ L Lavant-texte est «l'ensemble constitué par les brouillons, les manuscrits, les épreuves, les "variantes", vu sous l'angle de ce qui précède matériellement un ouvrage quand celui-ci est traité comme un texte. et qui peut faire système avec lui. (...) La difficulté vient de ce que le texte n'est pas le point d'aboutissement visé pendant la rédaction, mais un moment d'équilibrę; dès' lors qu'on envisage seulement un plus ou moins dinstabilité, où et quand commence cette décision qu'on appelle l'oeuvre et qui n'est pas un achèvement? De là l'intérêt d'un terme forgé sur le signe " -texte», et marqué par lui. On pose donc en principe que l'avant-texte est [dans] le texte et réciproquement."

Jean Bellemin-Noël: Le texte el lavant-texté. Cöll. "L». Paris. Larousse. 1972. p. 15.

\> Dans la dernière phrase, le mot mis entre crochets l'est par J. Bellemin-Noël.

«|... on classera schématiquement trois matériaux (ou trois hypostases des "documents de rédaction"), trois traitements particuliers, trois opérateurs spécifiques et trois ordres de finalité ou de compétence. On aboutira à trois phrases synthétiques du genre de celles-ci: - le manuscrit est un ensemble de supports matériels portant du texte, qui sont fixés-reproduits par un conservateur pour assurer l'authenticité d'un écrit et en faire l'objet d'un culte;

- les brouillons sont l'ensemble des documents qui ont servi à la rédaction d'un ouvrage, transcrits-présentés par un historien de la littérature en vue de reconstituer la préhistoire de cette réalisation tant du point de vue formel que du point de vue des contenus;

- un avant-texte est une certaine reconstruction de ce qui a précédé un texte, établie par un critique à l'aide d'une méthode spécifique, pour faire l'objet d'une lecture en continuité avec le donné définitif.

Ainsi se trouvent représentés nos cas de figure: un collecteurcollectionneur de mánuscrits, un éditeur,de brouillons et oeuvres 
complètes, un lecteur d'avant-texte ultra-spécialisé. Il va de soi qu'aucune séparation n'existé ențre ces trois démarches, qu'un même individu les adoptera successivement ou simultanément, avec des variations d'intensité.»

Jean Bellemin-Yoël: "Reproduire le manuscrit, présenter les brouillons, établir un avant-texte". Littérature. Paris, no 28 (no intitulé Genèse du texte), décembre 1977, p. 9.10.

"La valeur du préfixe "avant» exige quelques mots d’éclaircissement. II ne s'agit dans la logique de ma position ni de lui faire endosser une sorte de primitivité, au sens où l'antérieur serait une ébauche maladroite [...], ni de charger l'àntécédent d'un caractère inaugural lui conférant le prestige dune intuition première, repérable à l'état natif, que la suite du travail se contenterait d'embellir: ce qui est avant le texte publié, c'est déjà du texte et c'est déjà le texte. L'imparfait du texte nest pas privé de structuration, il peut être lu; il ne dit pas la mème chose que le texte au futur dans d'autres termes; on peut voir en lui un texte au conditionnel ou au subjonctif, mais il faut le traiter dans son présent.»

Jean Bellemin-Noël: "Avant-texte et lecture psychanalytique", dans Louis Hay et Peter Nagy (sous la direction de): Avant-iexte. texte après-texte. Colloque intemational de lexlologie |Matrafüred. octobre 1978|, Paris, CNRS et Budapest. Akadémiai Kiado. 1982. p. 162.

«|... i il a fallu opposer à l'indifférencié des brouillons, matériau disponible à l'historien sous lạpannière d'une factualité objective, le singulier d'un avant-texte produit à la fois théorique et subjectif formé, informé et transformé pạr les poșitions d'écoute propres à chaque critique."

Jean Bellemin-Noël: "En gúise de pọstface; l'essayage infini", Litterature, Paris, no 52 (no intitulé L inconscient dans lavant-texte), decembre 1983. p. 123, note 1.

EX. - L'ensemble du livre déjà cité (Le texte et l'avant-texte) est une analyse de 75 feuillets des brouilloñs d'un poème d'O. $V$. de LubiczMilosz.

$+\ll|\ldots|$ l avant-texte fonctionne aussi comme un * paratexte, dont la valeur (entre autres) de comminentaire et donc de * métatexte, par rapport au texte définitif, "est aussi évidente qu embarrassante, puisquil nous renseigne souvènt trës clairement (par exemple dans les esquisses de James) súr des intentioṇs. ou interprétations peut-être provisoires, et complètement abandonnées au moment de la rédaction définitive.»

Gérard Genette: Palimpsestes. La litteratureau second degré. Coll. «Poétique», Paris, Seuil, 1982, p. 447, п. 2. $\because " \cdot$ 


\section{Après-texte}

RÉF. - ? - Louis Hay et Peter Nagy (sous la direction de): Avanttexte, texte, après-texte. Colloque international de textologie [Matrafüred, octobre 1978], Paris, CNRS et Budapest, Akadémiai Kiado, 1982.

>> Sauf erreur, ce terme n'apparaît pas dans le cours des communications. Seule la dernière communication - celle de Winfried Woesler, intitulée "Des modifications textuelles prises comme interaction entre auteur et public» - parle de "variantes tardives" ( $p$. 208). Comme il semble que le titre du colloque, en 1978, soit devenu le sous-titre des actes publiés dudit colloque en 1982, il faut en déduire que ce terme, fait à l'évidence sur * avant-texte, s'est imposé aux éditeurs entre ces deux dates.

DÉF. - "Un dernier type d"* avant-textes consiste (...) en révisions et corrections apportées à un texte déjà publié; c'est la raison pour laquelle je parle ici d' "après-textes", mais il va de soi que cet après d'une édition est (ou se propose d'être) l'avant d'une édition ultérieure.»

Gérard Genette: Seuils. Coll. "Poétique», Paris, Seuil, 1987, p. 367.

EX. - André Gervais: «Le Vaisseau d'or»: texte et après-textes. Codicilles", Protée, Chicoutimi, vol. 15, no 1 (no intitulé Archéologie de la modernité. Art et littérature au Québec de 1910 à 1945), hiver 1987 , p. 31-43. Cet article analyse les 6 après-textes dudit poème d'E. Nelligan.

\section{Transtextualité}

RÉF. - Gérard Genette, 1979

DÉF. - «Mais il est de fait que pour l'instant le texte (ne) $m$ intéresse (que) par sa transcendance textuelle. savoir tout ce qui le met en relation, manifeste ou secrète, avec d'autres textes. J'appelle cela la transtextualité (...).»

Gérard Genette: Inroduction à larchitexte. Coll. «Poétique», Paris, Seuil, 1979, p. 87.

«ll me semble aujourd'hui (13 octobre 1981) percevoir cinq types de relations transtextuelles, que jénumérerai dans un ordre approximativement croissant d'abstraction, d'implicitation et de globalité lvoir, dans cet ordre, ${ }^{*}$ intertextualité, ${ }^{*}$ paratextualité, ${ }^{*}$ métatextualité, ${ }^{*}$ hy- 
Gèrard Genette: Palimpsestes. La littérature au second degré. Coll. «Poétique», Paris. Seuil, 1982, p. $8|-12|$.

\section{Transtextuel}

\section{RÉF. - Jean Ricardou, 1982}

DÉF. — «lci commence l'escalade vers les zones du vertige textuel. L'insertion du * métatextuel dans le texte, en effet, comporte plusieurs échelons.

Le premier, c'est la * métatextualisation du texte. En acceptant de sy faire théoricien, le praticien réussit la * métatextualisation de son texte. Bref, il devient autre (théoricien), et davantage lui-même (praticien), puisquil arrive, ce faisant, avec le troisième degré de l'autoreprésentation, à fournir au texte une complexité accrue. En acceptant de s'en faire praticien, le théoricien parvient à écrire le * texte-objet de sa * métatextualisation. Bref, il se fait autre (praticien), et davantage lui-même (théoricien), puisqu'il accède à une part de son impensé: le problème du manque de ce qu'il ajoute.

Le second degré, c'est la *textualisation $d u{ }^{*}$ métatextuel. Hors son objet, l'écrit théorique, en tant que * métatextuel honteux, possède un statut d'extra-territorialité ou, pour être plus exact, $d^{* *}$ extratextualité. En revanche, sitôt inscrit dans son * texte-objet, le * métatextuel court la chance de subir, à revers, de la part du * texte-objet, une incisive * textualisation. L'on propose dappeler transtextuel tout * métatextuel interne textualisé par son objet.

|... Avec lui, la représentation subit une double mise en crise: d'une part, celle des faits concrets, en tant qu'il est " métatextuel; d'autre part, celle de sa propre pensée à ce propos, en tant qu'il est * métatextuel textualisé. (...) le * transtextuel est le résultat superlatif du procès d'écriture."

Jean Ricardou: «L'escalade de l'autoreprésentation". Texle. Toronto, no 1 (no intitulé Lautoreprésentation. Le texte et ses miroirs), 1982, p. 19-20 et 23.

>>> On aura noté que ce terme, adjectif de «transtexte» (terme non actualisé dans les textes que nous avons consultés), est ici, selon le sens donné par J. Ricardou, un substantif à part entière. D'où la nécessité de lui consacrer une entrée distincte. On aura également noté que l'auteur utilise le substantif * métatextuel selon le sens donné à ce terme par B. Magné. 


\section{Architexte, architextualité, architexture}

\section{RÉF. — Louis Marin, 1974; Gérard Genette, 1979}

DÉF. - «L'utilisation d'une terminologie kantienne [...] est significative. Elle veut dire: 1/ que le monde naturel, comme lensemble spatio-temporel organisé et structuré par la perception constitue le texte primitif, l'architexte, de tout discours possible, son "origine" et son milieu d'instauration; $2 /$ que ce discours n'est autre que cet architexte saisi dans sa structuration dont les limites indépassables sont celles du monde naturel comme espace et temps perçus et dont les moyens sont ceux de l'activité linguistique; $3 /$ qu'en ce sens, le discours est la condition de possibilité de toute connaissance objective, scientifique - de tout * métatexte - et que celui-ci n'est autre que la restructuration du texte primitif du monde naturel.»

Louis Marin: "Pour une théorie du texte parabolique», dans Claude Chabrol et Louis Marin: Le récil évangélique. Coll. "Bibliothèque des sciences religieuses". Paris. Aubier, Cerf, Delachaux et Desclée de Brouwer, 1974, p. 167-168.

$\S$ «|... cette relation dinclusion qui unit chaque texte aux divers types de discours auxquels il ressortit. Ici viennent les.genres, et leurs déterminations déjà entrevues: thématiques, modales, formelles, et autres (?). Appelons cela, comme il va de soi, l'architexte. et architextualité, ou simplement architexture...»

Gérard Genette: Introduction à larchitexte. Coll. "Poétique", Paris, Seuil, 1979. p. 87-88.

"Le cinquième type [de relations * transtextuelles], le plus abstrait et le plus implicite, est l'architextualité l...). II s'agit ici d'une relation tout à fait muette, que n'articule, au plus, qu une mention * paratextuelle (titulaire, comme dans Poésies, Essais, le Roman de la Rose. etc., ou, le plus souvent, infratitulaire: l'indication Roman, Récit, Poèmes, etc., qui accompagne le titre sur la couverture), de pure appartenance taxinomique. Quand elle est muette, ce peut ètre par refus de souligner une évidence, ou au contraire pour récuser ou éluder toute appartenance. (... A la limite, la détermination du statut générique d'un texte n'est pas son affaire, mais celle du lecteur, du critique, du public, qui peuvent fort bien récuser le statut revendiqué par voie de * paratexte: ainsi dit-on couramment que telle «tragédie» de Corneille n'est pas une vraie tragédie ou que le Roman de la Rose n'est pas un roman. Mais le fait que cette relation soit implicite et sujette à discussion $|\ldots|$ ou à fluctuations historiques $\{\ldots \mid$ ne diminue en rien son importance: la perception générique, on le sait, oriente et détermine dans une large mesure l' "horizon d'attente» du lecteur, et donc la réception de l'oeuvre.»

Gérard Genette: Palimpsestes. La littérature au second degré Coll. "Poétique». Paris. Seuil, 1982, p. 11. 
>> L'objet de la poétique, dans le premier livre ici cité de G. Genette, est l'architextualité; dans le second, cet objet devient la * transtextualité. Ajoutons que c'est dans ce dernier livre que G. Genette s"avise un peu tard» que L. Marin a déjà proposé une définition du terme "architexte»; cette définition lui parait «[pllus près, en somme, de ce que je vais nommer * hypotexte" (Palimpsestes, p. 7). Ajoutons également que le même terme a été utilisé dans une communication (faite en juillet 1977) de Mary Ann Caws: «Le passage du poème: interrogation du seuil» (Cahiers de l'Association internationale des études françaises, Paris, no 30, mai 1978, p. 236); G. Genette remarque avec justesse (Introduction à l'architexte, p. 88) que M. A. Caws utilise ce terme «dans une toute autre acception, qui [lui\} échappe».

\section{Paratexte, paratextualité}

RÉF. - Gérard Genette, 1982

DÉF. - "Le second type [de relations * transtextuelles] est constitué par la relation [...] que, dans l'ensemble formé par une oeuvre littéraire, le texte proprement dit entretient avec ce que l'on ne peut guère nommer que son paratexte: titre, sous-titre, intertitres; préfaces, postfaces, avertissements, avant-propos, etc.; notes marginales, infrapaginales, terminales; épigraphes; illustrations; prière d'insérer, bande, jaquette, et bien d'autres types de signaux accessoires, autographes ou allographes, qui procurent au texte un entourage (variable) et parfois un commentaire, officiel ou officieux, dont le lecteur le plus puriste et le moins porté à l'érudition externe ne peut pas toujours disposer aussi facilement qu'il le voudrait et le prétend." Gérard Genette: Palimpsestes. La littéralure au second degré. Coll. «Poétiquè, Paris, Seuil, 1982, p. 9.

"Le paratexte est donc pour nous ce par quoi un texte se fait livre et se propose comme tel à ses lecteurs, et plus généralement au public. Plus que d'une limite ou d'une frontière étanche, il s'agit ici d'un seuil, ou - mot de Borges à propos d'une préface — d'un «vestibule» qui offre à tout un chacun la possibilité d'entrer ou de rebrousser chemin. "Zone indécise» [C. Duchet] entre le dedans et le dehors, elle-même sans limite rigoureuse, ni vers l'intérieur (le texte) ni vers l'extérieur (le discours du monde sur le texte), lisière, ou, comme disait Philippe Lejeune, «frange du texte imprimé qui, en réalité, commande toute la lecture». $|\ldots|$

Le paratexte se compose donc empiriquement d'un ensemble 
hétéroclite de pratiques et de discours de toutes sortes et de tous âges que je fédère sous ce terme au nom d'une communauté d'intérêt, ou convergence d'effets, qui me paraît plus importante que leur diversité d'aspect (Voir * péritexte et * épitextel."

Gérard Genette: Seuils. Coll, «Poétique», Paris. Seuil, 1987, p. 7-8 [10-11].

>> Dans Introduction à larchitexte (1979), il existe une toute autre définition du terme (p. 87); ce n'est que dans Palimpsestes qu'apparait la définition actuelle.

EX. — L’ensemble de Seuils est une analyse détaillée du paratexte.

\section{In-texte}

RÉF. - Raymonde Debray-Genette, 1977

DÉF. - «La poétique, en s'attachant à analyser des textes achevés, c'est-à-dire constitués en oeuvres, semblait avoir oublié la vieille génétique, réduite, il est vrai, le plus souvent à l'étude des «sources», ou plutôt avoir esquivé les problèmes véritables que soulèverait une génétique pleine et entière. [...) Mais leur antinomie mème devait aboutir nécessairement à cette confrontation entre génétique et poétique. Entre l'étude du * hors-texte comprise comme l'exploration des alentours, et celle, acharnée, de ce qu'on voudrait appeler l' «intexte», fermé sur lui-même, il existe une étendue, plus ou moins considérable, qui ne se réduit ni àl'un ni à l'autre, et dont on ne sait s'il faut l'appeler un texte, ni même du texte. Les manuscrits, au sens large du terme, forment ce pont de l'écriture, dont il n'est pas certain que nous sachions ce quil relie, origine ou fin."

Raymond Debray-Genette: "Génétique et poétique: esquisse de méthode". Littérature. Paris, no 28 (no intitulé Genèse du texte), décembre 1977, p. 19-20.

>> On remarquera que le terme ici utilisé, synonyme de texte, est créé pour les besoins de la cause, en tant que terme antinomique de * hors-texte. Entre l'in-texte et le hors-texte, prend place ce que J. Bellemin-Noël appelle l* avant-texte, un trajet qu'un lecteur propose dans les manuscrits au sens large.

\section{Hors-texte}

RÉF. - louri Lotman, 1964; Jean Bellemin-Noël, 1972 


\section{DÉF. - Voir * extratexte selon I. Lotman.}

$\S$ «[...] sortir de la problématique du texte, en même temps que de la sphère du poème, pour entrer résolument dans ce qu'on appellerait volontiers un hors-texte. C'est-à-dire repérer tout ce qui dans ces écrits renvoie à l'écrivain en tant qu'il est quelqu'un d'autre que l'auteur de ce poème (quelquiun d'autre, et aussi le même). Ce hors-texte ne visera pas une mise en relation avec les " contextes sociohistoriques et culturels (...).»

Jean Bellemin-Noël: Le texte el l avant-texte. Coll. «L", Paris, Larousse, 1972, p. 133.

$\gg>$ Voir ${ }^{*}$ extratexte et ${ }^{*}$ intertexte, tous deux selon J. BelleminNoël.

>> De tel * hypotexte, des passages peuvent, évidemment, ne pas ètre retenus, peu importent les raisons, par l* hypertexte; ces morceaux, du point de vue de l"* hypertexte, bien sûr, deviennent du hors-texte.

\section{Extratexte, extratextualité}

\section{RÉF. — louri Lotman, 1964; Jean Bellemin-Noël, 1972}

DÉF. - « $\dot{A}$ chaque niveau et pour toute la structure dans son ensemble, la somme des éléments artistiques réellement utilisés et des éléments extratextuels (rappelons que chaque élément par luimême représente une certaine relation) constitue la valeur universelle dudit niveau ou de la structure dans son ensemble.

II est tout à fait évident que la valeur artistique réelle des éléments textuels n'est compréhensible que par rapport aux extratextuels, une même quantité d'éléments textuels rapportée à différentes structures extratextuelles produit un effet artistique différent.

Seul le rapport de l'ensemble des éléments artistiques de tous niveaux, dans leur interconnexion et leur rapport à tout l'ensemble des éléments et liaisons extratextuels peut être considéré comme une description complète de la structure d'une oeuvre donnée. (...)

Pour nous faire voir à quel point changent les oeuvres dont le texte invariant est "inséré» dans différentes constructions extratextuelles, il suffit de prendre connaissance des nombreuses données concernant la réinterprétation par un lecteur étranger, ignorant, complètement, ou en partie, la structure de conscience (les «idées») et la vie pratique d'un peuple pour lui fort lointain, lorsqu'il lit des traductions textuellement exactes.

|...। Sans liaisons extratextuelles le texte devient — esthétiquement 
parlant - une oeuure en langue étrangère. C'est ce qui commande en particulier le phénomène, bien connu en histoire littéraire, de la mort et de la résurrection de l'efficacité poétique du texte. (...) Pour que tel ou tel "procédé» puisse devenir littérairement expressif, il doit auparavant exister comme potentiel dans l'ensemble de possibilités et d'impossibilités, de tolérances et d'interdits dont, à la suite de l'acte créateur, se détachent le texte et la paŕtie extratextuelle. Les deux sont indispensables pour la perception de l'art."

louri Lotman: "Le "hors-texte". Les liaisons extratextuelles de l'oeuvre poétique» (extrait d'un livre publié en langue russe en 1964l. Change. Paris, no 6. 3e trimestre 1970, p. 68-70.

>> Le terme "extratextuel» utilisé ici vient de "vnétekstovye, de vné-teksta, littéralement: hors-texte. (Aucune valorisation idéologique, ici. autour de l'objectif "textuel".)" (Change, p. 68, n. 1). Voir également, au sujet de ce terme tel qu'utilisé ici, les p. 127-128 de l'article de $M$. Angenot cité à * intertexte.

$\S$ «Autour de l'ensemble texte ${ }^{*}$ avant-texte [...), on considérera deux secteurs où l'on peut parler d'un intérêt de Milosz. Dans son intérêt d'abord, pour le mieux connaître, on essaiera de mettre au jour les informations qu'apportent ces pages manuscrites; cette opération peut ètre entreprise sous le nom d'étude de lextra-texte, en tant qu'elle procède d'une extraction (ce qu'on sort de l'* avant-texte pour le rapporter à Milosz). D'autre part, on envisagera la place que tiennent ces pages dans l'ensemble de l'oeuvre, les échos qu'elles y provoquent ou qu'elles en reçoivent: cela fera l'objet d'une recherche de leur intersection avec les autres oeuvres de Milosz, quion peut baptiser $l^{*}$ inter-texte. (...)

En regardant les fragments laissés de côté ldans la formation de l'* avant-texte], on découvre un certain nombre de "documents": des morceaux pratiquement rédigés et abandonnés définitivement, des bribes qui seront réutilisées ailleurs, des renseignements dordre biographique involontairement procurés. Leur point commun et l'intérêt de leur apport, c'est qu'ils permettent de mieux cerner l'univers intellectuel de Milosz, en quelque sorte indépendamment de la rédaction Idu poème intitulél la Charrette.»

Jean Bellemin-Noël: Le texte et lavant-texte. Coll. «L», Paris, Larousse, 1972. p. 133-134.

EX. - Les p. 134-138 du livre ici cité. 